\title{
FORMACIÓN DE FORMADORES. EXPERIENCIA EN EL SALVADOR
}

\author{
Paloma González Aguadol
}

\section{Resumen}

El siguiente artículo presenta una sintesis de las actividades realizadas dentro del Convenio Universidad Autónoma de Madrid (UAM), la Universidad Nacional de El Salvador (UES) y la Universidad Centroamericana (UCA), cuya intención era buscar la transformación de los y las docentes en ejercicio. Las redes de conocimiento y relación generadas entre los docentes participantes y su impregnación de estas modalidades de trabajo han motivado cambios institucionales.

Palabras claves: formación docente, comunidad rural, trabajo interuniversitario.

\section{Abstract}

The present article is a synthesis of activities developed as a part of the agreement between Universidad Autónoma de Madrid (UAM), Universidad Nacional de El Salvador (UES) and Universidad Centroamericana (UCA), which goal was to favor active teacher's transformation. As a result of the activities and the relation generated between participants, knowledge networks were developed and institutional changes have occurred.

Keywords: teacher training, rural community, interuniversity work.

\section{Antecedentes}

\section{Situación del país en la época en que la UAM suscribió el convenio}

$\mathrm{E}$ Salvador es el país más pequeño de Centroamérica (el Pulgarcito, le llamaba su poeta Roque Dalton). Como casi todos recordamos, fue protagonista doloroso de las noticias en el último tercio del siglo XX. Tras una década de preguerra con masacres constantes, en 1980, se reconoció oficialmente la

Universidad Autónoma de Madrid. Profesora titular (Dra.), Facultad de Profesorado y Educación. CORREO ELECTRÓNICO: paloma.gonzảlez@uam.es

Recibido: 3 de noviembre-2006 - Aprobado: 30 de novicmbre-2006 
situación de guerra civil, que duró hasta la firma de los Acuerdos de Paz (enero de 1992), aunque el desarme fuera un año después.

Igual estaba clara la colaboración militar de los EE. UU. en la guerra (1980-92); todos los salvadoreños han tenido oportunidad de conocer voluntarios de España o de México y estos dos países ejercieron como mediadores de los Acuerdos de Paz, texto en que son citados como "los países amigos" por ambas partes contendientes.

\section{La realidad universitaria en el período: nuestro convenio}

La Universidad Nacional de El Salvador vivió todo tipo de violencias, cierres, destrozos de instalaciones, períodos de ocupación militar y, por supuesto, asesinatos y detenciones de académicos, docentes y estudiantes. El acoso fue tan fuerte, que el lema todavía escrito en algunas paredes del campus es "LA UES SE NIEGA A MORIR".

Otra vía de acoso oficial fue la autorización masiva para que quien lo solicitara, fundara una universidad. Esto hizo que un país pobre, con SEIS MILLONES de habitantes, tuviera autorizadas unas 50 universidades privadas al terminar el conflicto. Dado el caos académico vivido, en 1995, hubo necesidad de regular los requisitos de estas instituciones improvisadas para ser reconocidas como universidades (iy para completar la validez de los títulos ya expedidos!).

En esas fechas, un compañero de la UAM, que ahora está en la Politécnica de Madrid, promovió el actual Convenio de la UAM con las universidades UES y UCA.

Si la situación de exilio y deterioro continuado por más de 20 años (1970-1992) generó la consiguiente descapitalización intelectual en el país, que padecen todas las universidades, esta Universidad se ha tenido que reconstruir hasta físicamente, con su presupuesto congelado desde los años 70 y los edificios derruidos por la guerra y los terremotos. También, ha sido importante el esfuerzo de reconstrucción humana a partir de su deficiencia en los recursos humanos y de la conflictividad y tensión vivida entre ellos.

La política seguida en esta Universidad ha sido priorizar la organización de posgrados para sus propios docentes en el ejercicio, lo que ha dado como resultado la mejora institucional.

La UES es la única universidad pública, e imparte la docencia en las distintas zonas del país; cuenta con unos 40.000 estudiantes. 
Por otra parte, si siempre la gestión universitaria es un tanto tediosa, evidentemente las circunstancias descritas relativizan toda actuación. La reflexión respecto a que la falta de capacidad de gestión, es una carencia tan importante como cualquier otra, permitió trabajar por encima de las dificultades. El regreso de docentes exiliados o formados en otros países y la continuidad en la Cooperación va ayudando, poco a poco, a superar esas limitaciones.

Su tradición de elección democrática de cargos académicos, también genera el inconveniente de colapsar cada 4 años la gestión y romper la continuidad de planteamientos (en estos años, he tenido tiempo de conocer a cuatro equipos electorales).

En su calidad de privada, tiene un alumnado reducido. Su cantera de personal docente es común con las otras universidades; la diferencia con la anterior, es que utiliza las aportaciones voluntarias de profesores universitarios de otros países para incrementar su oferta de posgrados para captar estudiantes.

La UCA tuvo una mayor dificultad para reestructurarse después de la guerra, porque no contó con docentes nacionales, sino extranjeros. Cuando casi al final de la guerra mataron a 6 jesuitas de sus cuadros directivos ( 5 de origen español), fue posible
La Universidad Centroamericana (UCA) es la universidad privada de los jesuitas, una de las primeras universidades privadas autorizadas. Ha conseguido fama de ser la segunda universidad en prestigio del país, después de la UES. En territorio español, resulta ser una de las más conocidas, pues sus cuadros directivos, de procedencia española, han sabido informar sobre ella.

sustituirlos igualmente desde España y sigue el mismo equipo. Por supuesto, hay carencias de gestión que han retrasado un poco su desarrollo, si se compara con la UES.

\section{Inicio de las actividades}

Con ocasión de los Acuerdos de Paz (enero de 1992), el profesor que había promovido el Convenio ejercía como su tutor, y cursó una petición al profesorado de la UAM para realizar labores formativas en las universidades indicadas, en calidad de docentes voluntarios. 
Como profesora titular de la Facultad del Profesorado y Educación de la Universidad Autónoma de Madrid, tuve conocimiento de esta situación y me ofrecí a participar en el marco académico del Convenio; como en ese momento no se disponía de presupuesto suficiente para costear los pasajes de todas las personas, el financiamiento corrió por cuenta propia.

Desde ese año (1992), he ido respondiendo a las demandas planteadas por las universidades contraparte, por lo que compatibilizo mi carga docente en la Facultad, con trabajo realizado en mis vacaciones y visito El Salvador; esto me ha permitido colaborar tanto con la UCA como con la UES. En ambos casos, el trabajo se ha orientado hacia la formación de sus docentes en ejercicio, pensando que tenía mayor proyección para la reconstrucción de los pilares institucionales universitarios.

Con la realización de estos trabajos, me he especializado en metodologías para el diseño y realización de Proyectos de Mejora Educativa. En estos años, también, he colaborado con otras universidades de Latinoamérica con las que tiene Convenio la Universidad Autónoma de Madrid. En América Latina, la modalidad incluye el trabajo multiplicador en redes con efectos de cascada.

En la UAM, participo en el equipo que dirige Ana Rodríguez Marcos (actualmente constituido como equipo EMIPE) que lleva esta línea de trabajo respecto a la práctica de la Facultad. Este equipo ha sido reconocido por sus variados proyectos de innovación. También he participado en el equipo dirigido por Asunción González-Yerro para el Área de Psicología de la Educación.

\section{Esbozo de algunas líneas de actividad desarrolladas que muestran la continuidad con las acciones actuales}

El trabajo que se va a comentar se ha realizado más intensamente con la Universidad Nacional, que se ha encargado, especialmente, de la transformación de sus propios docentes en ejercicio. Se han elegido actividades de amplia proyección que están sirviendo para potenciar los recursos humanos existentes en las mismas realidades y que necesitan desarrollarse; este trabajo se proyecta en distintos niveles: la propia institución, estudiantes y actores comunales.

En los difíciles años de los Acuerdos de Paz, se le hicieron diferentes encargos que tenían que ver con la actualización de docentes en ejercicio. La peculiar situación que se vivía en esos años se pudo aprovechar para diseñar y ensayar experiencias de transformación de la institución y de su Proyecto Social a través del reducido número de profesores a los que tenía acceso (poco más de 1000). 
Las innovaciones se realizaron a través de la formación teórico-práctica diseñada y de la metodología de trabajo aplicada. Como producto final, la dirección y asesoría de trabajos de investigación (en algunos casos, las tesis de maestría de casi 20 docentes) permitieron el acompañamiento y seguimiento de proyectos contextualizados, que les ha ayudado a diseñar y elaborar aplicaciones concretas de amplio impacto para la transformación, tanto de cada Facultad como de las comunidades destinatarias de las acciones.

Para realizar estas tareas, hubo que generar un equipo y se invitó a docentes tanto de la UAM como de distintas universidades españolas, que colaboraban con la financiación de los pasajes de su profesorado.

De entre las actividades realizadas entre 1992 y 2006, en el marco del Convenio UAM-UES-UCA, se enuncian a continuación algunas que tienen especial relación con demandas actuales:

a. Maestría para actualización del profesorado en ejercicio (UES)

Entre otras actividades realizadas en la Universidad Nacional de El Salvador, está el diseño, dirección y coordinación de la Maestría en Formación para la Docencia y Proyección Universitaria dirigida a la actualización de los cuadros docentes de Facultades, preferentemente, Sanitarias e Ingenierías, tras los difíciles años de la guerra civil.

Aunque las relaciones empezaron en 1992 con los Acuerdos de Paz, este proceso se formalizó en 1994 con las metodologías indicadas anteriormente. Los egresados en 1996 culminaron su proceso en 1998 y 1999 con la presentación pública y defensa de Tesis de Maestría, que sistematizaban las transformaciones conseguidas, innovando en metodologia docente y diseño de aplicación de los conocimientos a la mejora del contexto.

b. Iniciación de equipos de investigación educativa (UES)

En estrecha colaboración con el Instituto de Formación y Recursos Pedagógicos (INFORP) de la UES, se ha iniciado en metodología de Investigación-Acción, a distintos equipos de docentes del Profesorado Universitario de las Delegaciones Regionales de la UES, en todo el país, para la formación de maestros urbanos y rurales.

c. Formación de formadores para mamás que ejercen de educadoras en comunidades sin centros infantiles (UCA)

Simultáneamente, y debido al interés mostrado, se inició este tipo de formación con la UCA, y se han compartido estas actividades con el 
Programa de Atención a Educadoras Infantiles (PAEI) para la formación de educadoras infantiles (internacionalmente, también denominadas madres cuidadoras, casi sin estudios primarios que están al frente de centros infantiles). Aunque el Programa era financiado por la ONG Belga Van Leer, durante los años de su realización, tuvo sede en la UCA.

Entre otras actividades mediante la misma metodología de diseños de multiplicación en cascada, se formó, durante varios años, a un grupo de 12 formadores propios del Programa llamados promotores o facilitadores que realizaron la formación directa de casi cien mamás cuidadoras, diseminadas en comunidades semiurbanas o rurales. También se formaron a 30 formadores de instituciones locales, del Instituto Salvadoreño de Protección al Menor y de los Hogares Infantiles SOS.

Todas estas experiencias han sido especialmente útiles para adquirir la preparación necesaria para el contacto directo con las personas de distintas comunidades rurales y semirrurales del país.

\section{Algunos logros generados por la sostenibilidad interna del trabajo, ocho años después de terminado el programa}

Como uno de los objetivos básicos de los trabajos realizados, estaba la transformación de la Metodología docente y de la Proyección Social a través de los equipos que iban constituyendo las personas que se formaban. Puede constatarse como logro que en los Departamentos y Facultades en que hay personas que asistieron a estas actividades, se siguen realizando actividades de coordinación para la Innovación Docente, cada cual en la línea específica de su Facultad.

Los logros posteriores de equipos participantes en nuestros programas permiten visualizar la amplia proyección que, con el paso de los años, han ido teniendo los equipos constituidos a partir de este reducido grupo de docentes. Las redes de conocimiento y relación generadas entre los docentes participantes y su impregnación de estas modalidades de trabajo han motivado cambios institucionales a escala micro y les ha llevado a seguir dinámicas similares para proyectarse en red con: 


\section{a) Redes internas articuladas con instituciones de ámbito interna- cional}

Al grupo de docentes de la Facultad de Medicina que cursó la Maestría, como aplicación del trabajo hecho en sus tesis, se le encargó generar un Departamento de Educación Médica, para diseñar y poner en marcha un plan de actualización en metodologías docentes para el profesorado de las siete carreras que se imparten en su Facultad, el cual se realiza con gran éxito.

En el 2006, formaron la tercera promoción, cuyos profesores se van incorporando a proyectos de mejora de su docencia.

En estos años, cuando consiguieron preparar una primera generación, la Universidad vio la necesidad de coordinar la formación docente de todas las carreras de salud (incluyendo las externas a Medicina, tales como Farmacia y Odontología). Para ello fundó su Centro de Investigación y Desarrollo de Salud de la UES (CENSALUD).

b) Redes de actores locales implicados en su propio desarrollo (docentes universitarios, estudiantes y comunidades rurales)

Del mismo modo, el grupo de docentes de áreas técnicas (Ingenierías y Arquitectura) continuó el trabajo transformador iniciado en su tesis. A través de la Proyección Social de sus estudiantes, realiza la aplicación de sus estudios acompañando Proyectos de Desarrollo Integral (desarrollo local y reconstrucción fisica y humana, reconciliación) en comunidades rurales y semiurbanas, lo que permite tener relación con una serie de lugares con diferentes niveles de organización comunal (desde la no vertebración a estadios avanzados de autogestión).

La Facultad de Arquitectura acompaña proyectos de autoconstrucción en comunidades de todo el país (deficitarias de vivienda popular en alrededor del $90 \%)$.

Los docentes de Ingeniería Agronómica orientan a sus estudiantes en la realización de un trabajo similar dentro de su especialización agrícola.

El encargado en la AECI de El Salvador, Francisco López, ha descubierto la valía del apoyo técnico (y confiable) que estas redes de colaboración le aportan y recurre a su mediación técnica cuando le ha sido necesario. 


\section{Redes internas articuladas con instituciones de ámbito internacional}

Por los resultados obtenidos, la Universidad Nacional de El Salvador (UES) ha sido elegida como subsede regional de la DUAL para Centroamérica (la sede está en Cuba) y se le ha encomendado un proyecto regional respaldado, entre otras instituciones; por: la Unión de Universidades de América Latina (UDUAL), la Organización Panamericana de la Salud (OPS) y el Instituto Superior de Ciencias Médicas de La Habana.

La propuesta es capacitar, en un período de diez años, a la mayor cantidad de docentes posibles de Ciencias de la Salud de todas las universidades afiliadas a la UDUAL.

Ante la envergadura del compromiso, las autoridades de la UES me han pedido la colaboración y fui invitada formalmente a participar "como Docente e Investigadora en el Proyecto, habida cuenta de los antecedentes de colaboración continuada". En principio, trabajé allí seis semanas durante el verano de 2005, con el objeto de asesorar en la planificación y revisión de los contenidos y como inicio de posteriores solicitudes que se pudieran programar.

Esto permitió la constitución de un equipo mixto para la investigación del impacto de los cambios realizados en la práctica por los docentes de las áreas de salud de la Universidad y mantener la dinámica de su propio perfeccionamiento.

\section{Redes de actores locales implicados en su propio desarrollo}

En este ámbito, se intenta dar el salto cualitativo de generar equipos mixtos de trabajo en investigación que desarrollen, de manera interdisciplinaria, redes conjuntas entre docentes de universidades latinoamericanas y españolas.

Para tal fin, se ha presentado a la $3^{\text {a }}$ Convocatoria de concesión de ayudas para PROYECTOS DE INVESTIGACIÓN UAM-SCH para la cooperación con América Latina el Proyecto de Investigación-Acción Diseño, intervención y evaluación de un programa para el Desarrollo Rural que sirva de Modelo de actuación interdisciplinaria implicando a expertos universitarios de las diferentes áreas, fortaleciendo las capacidades del tejido social en las zonas de incidencia para potenciar su sistema productivo y su reconstrucción 
integral (fisica, humana y social) en municipios de El Salvador especialmente derruidos por el terremoto.

Para esta actividad, se ha constituido un equipo interdisciplinario abierto a las participaciones que en la misma línea de trabajo se puedan articular.

\section{Nuevas demandas surgidas durante esa estancia (2005)}

El trabajo presentado es modesto y está limitado por el escaso tiempo de que se dispone. Con todo, distintas instancias de la Universidad Nacional de El Salvador(UES), manifestaron su interés en que se les ayude a organizar la forma en que el conjunto de sus actividades docentes se articulen conforme al Modelo de Transformación diseñado. Es decir, integrando la mejora en los tres ejes que, en su planteamiento, son fundamentales: Docencia, Investigación y Proyecto Social.

\section{Consejo de investigaciones científicas}

Tras estos años de reconstrucción postbélica, la Universidad Nacional de El Salvador quiere retomar su capacidad investigadora interrumpida por estos 30 años de crisis. Para ello, ha creado recientemente su Consejo de Investigaciones Científicas (con financiación oficial de España).

Debido a la descapitalización intelectual del país (y de su Universidad) en este periodo, han puesto a dirigir este Consejo de Investigaciones a una doctora salvadoreña formada en el extranjero (química-farmacéutica). Para su organización, ha valorado las distintas aportaciones que la Universidad ha recibido en estos años y, desde su formación estrictamente científica, nos ha pedido cooperación para la articulación de los incipientes equipos desde la Metodología utilizada.

En las distintas sesiones de trabajo conjunto, se propusieron los siguientes objetivos:

- Generar sinergias entre equipos mixtos interdisciplinarios de las distintas facultades, de forma que potencien su capacidad de impacto.

- Avanzar en el modelo de integración sus tres ejes (docencia, investigación y proyección social).

- Favorecer procesos propedéuticos para que a mediano plazo sea posible, con éxito, la implantación de doctorados. 


\section{Vicerrectoría académica y dirección de postgrados}

El equipo rectoral, al evaluar los logros de las distintas maestrías impartidas en los años 90, ha solicitado un relanzamiento de la Maestría en Formación para la Docencia y Proyección Universitaria.

Esta petición la motivan en la trayectoria profesoral que han mostrado egresados de distintas maestrías, en los años transcurridos desde su acreditación. Advierten una gran diferencia entre los docentes que cursaron su postgrado en programas que pueden seguir repitiendo a sus estudiantes (y cuya proyección terminó cuando se expidió el título a las personas), frente a la inquietud en renovar y cooperar con el desarrollo de la institución, que se advierte en los docentes que siguieron el programa dirigido desde la UAM.

En estos momentos en que la UES está necesitando despegar en la capacidad investigadora, concibe esta maestría como iniciación de equipos de trabajo que vayan generando una cultura de sistematización de mejoras y producción de un bagaje investigador que sirva como propedéutico para los grupos de investigadores más avanzados.

Con este fin se ha constituido una Comisión Académica para la actualización del Programa Interfacultativo de Maestría, con el objeto de mantenerlo como título propio de la UES, para el desarrollo de sus docentes, incorporándolos a practicar como miembros de equipos investigadores, que piden orientemos desde la misma Metodología.

Su meta sería generar un grupo capaz de iniciar un doctorado en esta línea metodológica de transformación y la mejora institucional que ya tiene un modelo en la UNED.

\section{Situación de la gestión al 2006}

Evidentemente, este conjunto de compromisos exceden lo que un equipo reducido de docentes de una Universidad pueden hacer compatible con los propios compromisos, por lo que sería deseable acudir a la coordinación con otras universidades o instituciones que, de forma voluntaria, quieran participar o ayudar económicamente, liberando de la carga docente a las personas que se impliquen.

Por otra parte, da pena que dentro de las distintas cooperaciones que la UAM puede estar realizando, no capitalice este esfuerzo que los receptores perciben como continuado, sistemático y con gran capacidad transformadora. 
Personalmente, siento la limitación de que el compatibilizar el trabajo en la UAM con gastar tanto tiempo y energías en la UES, no da la posibilidad de acumular lo que supondría la gestión de mejores medios o permisos para mayor efectividad en esta colaboración.

La Universidad contraparte sabe que este tipo de apoyo lo ha recibido siempre en forma multilateral, por lo que si alguna entidad oficial (nacional e internacional) tuviera intención de implicarse, le resultaría natural.

En el año 2006, se ha vuelto a formalizar una petición para que me desplace a realizar las tareas que ellos visualizan como específicas respecto a colaboraciones que reciben de otras universidades, en el sentido de que les sirve de impulso, consolidación y coordinación de los diferentes avances que como institución van consiguiendo.

Hasta ahora, tener un convenio marco abierto, permitía presupuestar (más o menos) los pasajes independientemente de las fechas en que la gestión de la Universidad contraparte haga llegar la invitación. Según la tutora del convenio con El Salvador, los requisitos de las convocatorias periódicas que realiza actualmente el Vicerrectorado de Relaciones Internacionales no permiten gestionar la financiación de viajes, cuando las invitaciones son cursadas con tan poco tiempo.

El convenio de la UAM ha dedicado siempre un presupuesto mínimo para esta actividad. Durante los años fuertes de docencia en la Maestría, sólo en una ocasión la UAM ha financiado pasajes para dos profesoras. Así por ejemplo he tenido que viajar dos veces al año con apoyo de la AECI e ingreso personal, para no romper la continuidad y compromiso de trabajo.

Entre las dificultades de este tipo de actividades están:

- Exige mucha motivación tanto para hacer un esfuerzo prolongado en los años, como para ir especializándose en unas tareas para las que se necesita añadir a nuestro desempeño habitual capacidades y competencias específicas, tanto en el tipo de análisis que se realizan como en el tipo de relaciones que se establecen.

- Hay una gran desproporción entre la gran dedicación y especialización que exige y la escasa o nula entidad que se le concede en el currículo docente de los profesores, lo que hace desistir a personas cuya aportación sería valiosa.

- Quizá por su novedad en nuestro país, supone un serio inconveniente de gestión su planteamiento diluido en la UAM: son tareas académicas 
e investigadoras, pero no se pueden incluir entre lo que se considera de alto nivel.

- $\quad$ Esto hace que en cualquiera de los Vicerrectorados en que se comenta pueden resultar actividades más o menos interesantes, pero parece que no se adecua totalmente a las prioridades de ese Vicerrectorado (Investigación, Académico, Innovación, Cooperación, Relaciones Internacionales...).

Es una lástima que con lo prestigiada que está esta línea de trabajo en la Universidad contraparte, la UAM (en Madrid) no haya tenido capacidad de capitalizar como propios este tipo de trabajos o incluso liderar una línea de cooperación con universidades coparticipantes.

Quizá el hecho de saber que esta actividad está abierta y sus peculiaridades metodológicas, sería suficiente para, al menos permitir organizar otro tipo de procedimientos de gestión de estos viajes, cuando no de abrir una línea de debate y reflexión sobre las aportaciones que realiza nuestro profesorado.

Es nuestro deseo, continuar con el desarrollo de este Convenio, para promover el aprendizaje y el trabajo interuniversidades. Las experiencias descritas pueden ser consideradas como exitosas. 


\section{REFERENCIA}

González, Paloma (2005). Informe del proyecto: Formación de formadores. Universidad Autónoma de Madrid. 